\title{
El consumo de sustancias adictivas en las Américas
}

\author{
Peruga, A. *; Rincón, A. * y Selin, H.* \\ Organización Panamericana de la Salud \\ Enviar correspondencia:
}

Armando Peruga. Asesor Regional de Tabaco, Alcohol y Drogas. Programa de Salud Mental. 525 23rd St. NW Rm 822 . Washington DC 20037 (EEUU)

\section{RESUMEN}

El consumo de tabaco, alcohol y otras drogas está ligado a cerca de una cuarta parte de las defunciones anuales que se producen en las Américas. En el Cono Sur de América Latina, a los 15 años de edad ya fuma cerca del $40 \%$ de los jóvenes y la gran mayoría respira en casa el aire contaminado por el humo de tabaco de los demás. En América Latina cada persona consume en promedio 6 kilos de alcohol puro por año, lo que constituye la cifra más alta del mundo menos desarrollado. Aunque los datos sobre el consumo de drogas ilegales no son abundantes sabemos que la marihuana es la droga que mayor proporción de la población consume. Se estima que 45 millones de ciudadanos de las Américas la consumen. Si bien la carga de enfermedad que genera el consumo de drogas ilegales no llega a la magnitud de la ocasionada por las drogas legales, las consecuencias sociales son mucho mayores.

Las respuestas de los países de las Américas al consumo de sustancias adictivas han sido insuficientes. Los principales desafíos para el control del tabaquismo y del uso de alcohol en las Américas son dos: disminuir la asequibilidad de los productos y evitar la exposición de la población a las prácticas comerciales de promoción de los productos de las industrias tabacalera y alcoholera. Además, en el caso del tabaco, hay que proteger a los no fumadores del humo de los demás.

En el caso de las sustancias ilegales, los resultados hasta ahora de un enfoque orientado al control de la oferta han sido limitados. El desafío es hacer hincapié en el control de la demanda, mediante intervenciones de prevención, desintoxicación, tratamiento y rehabilitación, así como de reducción de daño.

Palabras clave: epidemiología, prevalencia, droga ilegal, tabaco, alcohol, políticas sobre drogas, Latinoamérica

\section{SUMMARY}

The use of tobacco, alcohol, and other drugs is probably linked to nearly a fourth of annual deaths that occur in the Americas. In the Southern Cone of Latin America, close to $40 \%$ of teenagers already smoke by age 15 . The great majority of them breath at home air polluted with second hand smoke. In Latin America each person consumes 6 kilograms of pure alcohol each year on average, the highest figure in the less developed world. Despite the fact that data on use of illegal drugs is not widely available, it is known that marihuana is the drug of choice of a high proportion of the population. It is estimated that 45 million people consumes marihuana in the Americas. It is true that the disease burden generated by illegal drugs is not as high as that generated by legal drugs. However, their social consequences are higher.

The principal challenges for the control of smoking and of the use of alcohol in the Americas are two: diminish the availability and affordability of the products, and to avoid the exposure of the population to the marketing practices of the tobacco and alcohol industries. Furthermore, in the case of tobacco, it is necessary to protect non-smokers from the smoke of others.

In the case of the illegal substances, the results until now of an approach focused on supply control have been limited. The challenge is to emphasize the control of the demand through interventions of prevention, detoxification, treatment, and rehabilitation, as well as harm reduction.

Key words: epidemiology, prevalence, illegal drug, tobacco, alcohol, drug policies, Latin America
A Igunas sustancias adictivas en las Américas se han producido y consumido desde tiempos inmemoriales. La hoja del tabaco y la de coca son clásicos ejemplos de ello. Sin embargo, hasta épocas recientes su consumo nunca tuvo repercusiones de salud pública especialmente serias. Hoy en día, el consumo de tabaco, alcohol y otras drogas está ligado a cerca de una cuarta parte de las defunciones anuales que se producen en esta región del mundo. En las líneas que siguen se pretende establecer un panorama del consumo de dichas sustancias y sus consecuencias para la salud de los americanos, sean estos del norte, centro o del sur de este continente. 


\section{Mortalidad atribuible al consumo de tabaco}

El consumo de tabaco es la principal causa de muerte evitable en las Américas y en el mundo. Más de un millón de personas mueren por consumir tabaco cada año en las Américas, de los cuales $46 \%$ son mujeres. El Cuadro 1 muestra la mortalidad promedio anual atribuible al tabaquismo en esta región correspondiente al primer lustro de la década de los 90, período para el que existe la información de mortalidad más completa y actualizada para el conjunto de la región como para hacer una estimación confiable. La mitad de este millón de defunciones ocurre en América Latina. Las defunciones atribuibles al tabaquismo del Cono Sur representan un cuarto de todas las muertes estimadas de esta subregión, seguida de Norteamérica $(23,5 \%)$ y Brasil $(18,8 \%)$. Comparado con un estudio anterior ${ }^{1}$ la proporción de muertes causadas por el consumo del tabaco ha aumentado sustancialmente, si bien las cifras no son totalmente comparables debido a ligeras diferencias en la metodología utilizada.

\section{Cuadro 1. Mortalidad anual atribuible al tabaquismo en las Américas por subregiones, para el periodo 1990-94 y 1985}

\begin{tabular}{|lcr|}
\hline Subregión & \multicolumn{2}{c|}{ Número estimado de defunciones } \\
& Promedio anual 90-94 & 1985 \\
\hline América Latina & 500.427 & 98.100 \\
Área Andina & 94.911 & 8.200 \\
Brasil & 205.438 & 32.400 \\
Caribe Latino & 26.268 & 10.200 \\
Centroamérica & 24.675 & 900 \\
Cono Sur & 108.701 & 32.100 \\
México & 40.434 & 14.200 \\
Caribe & 5.601 & 1.900 \\
Norteamérica & 565.527 & 426.100 \\
\hline Total & 1.071 .555 & 526.000 \\
\hline
\end{tabular}

\section{La Prevalencia del Tabaquismo}

Las tasas de tabaquismo varían mucho en la Región de las Américas, registrándose las más altas en los países del Cono Sur, en particular Chile y Argentina [alrededor de $45 \%$ de los hombres y $35 \%$ de las mujeres] y las más bajas en algunos países del Caribe. ${ }^{2,3}$ Los datos de tendencia existentes indican que Canadá y Estados Unidos han mostrado reducciones importantes y sostenidas de la prevalencia de tabaquismo. ${ }^{2}$ La prevalencia de tabaquismo de la mayoría de los demás países de las Américas han permanecido relativamente estables con algunas excepciones.

La adicción al tabaco empieza generalmente en la adolescencia. En la mayoría de los países de las Amé- ricas, más del 70\% de los fumadores comenzaron a fumar antes de los 18 años. De hecho una proporción importante de jóvenes fuma antes de cumplir los 18 años. Recientemente un encuesta mundial ha permitido comparar por primera vez en América Latina y el Caribe, las actitudes, el conocimiento y el comportamiento de los jóvenes en lo referente al uso del tabaco. Es también la primera vez que datos comparables a nivel mundial y útiles para la toma de decisión en políticas estarán disponibles sobre el mercado clave de la industria tabacalera: los nuevos consumidores, casi exclusivamente jóvenes, que reemplazarán a los fumadores que mueren o dejan de fumar, para mantener o aun aumentar las ganancias de la empresa tabacalera. ${ }^{4}$ Al final del 2000, un total de 111 países y territorios del mundo tenían resultados disponibles o estaban aplicando la encuesta, entre estos 13 países latinoamericanos y 18 países del Caribe de habla inglesa. ${ }^{5}$ El Cuadro 2 presenta los datos de las Américas disponibles de la Encuesta Mundial de Tabaquismo en Jóvenes. Entre el 15 y el 30\% de los jóvenes de 13 a 15 años de la mayoría de los países de América Latina consumían tabaco en el momento de la encuesta. Al igual que en los adultos, las prevalencias más altas se observan en el Cono Sur. En el Caribe las cifras son más bajas, variando entre el 13 y $21 \% .{ }^{6}$ La mayoría de los jóvenes que comienzan a fumar se convertirán en fumadores empedernidos a pesar de querer dejar de fumar. En muchos países, más de dos tercios de los adolescentes fumadores intentaron dejar de fumar infructuosamente, debido a la adicción a la nicotina.

\section{Desafíos para la prevención de los problemas rela- cionados con el consumo del tabaco}

Los principales desafíos para el control del tabaquismo en las Américas son tres: disminuir la asequibilidad de los productos tabacaleros, evitar la exposición de la población a las prácticas comerciales de promoción de los productos de la industria tabacalera y proteger a los no fumadores de del humo de los demás. ${ }^{7}$ En algunos países como Argentina y Chile, entre el 60 y el $70 \%$ de los jóvenes entre 13 y $15 \%$ respiran a diario en su casa el humo de los fumadores. Estas cifras alcanzan valores de cerca del 90\% cuando se mide la exposición en cualquier ámbito de la vida diaria de estos jóvenes.

Sólo tres países de las Américas tienen restricciones amplias de la publicidad y patrocinio de los productos tabacaleros: Canadá, Cuba y Brasil. Como dato de ilustrativo de la agresividad del marketing de los productos tabacaleros, se sabe que entre un $10 \mathrm{y}$ $15 \%$ de los jóvenes latinoamericanos de 13 a 15 años de edad han recibido ofertas directas de cigarrillos gratis por personas que trabajan para la industria tabacalera, a pesar de que formalmente la propia industria 
Cuadro 2. Prevalencia de tabaquismo e intentos de dejar de fumar en jóvenes de 13 a 15 años en América Latina y el Caribe, 1999-2000

\begin{tabular}{|c|c|c|c|c|}
\hline \multirow[b]{2}{*}{ País } & \multirow[b]{2}{*}{ Localidad } & \multicolumn{3}{|c|}{ Proporción de jóvenes que } \\
\hline & & $\begin{array}{l}\text { Han fumado } \\
\text { alguna vez }\end{array}$ & $\begin{array}{c}\text { Fuman } \\
\text { actualmente }\end{array}$ & $\begin{array}{l}\text { Intentaron dejar de } \\
\text { fumar sin éxito }\end{array}$ \\
\hline \multicolumn{5}{|l|}{ América Latina } \\
\hline Argentina & Buenos Aires & 60,0 & 30,2 & 51,6 \\
\hline Bolivia & Cochabamba & 53,3 & 23,5 & 59,8 \\
\hline Bolivia & La Paz & 55,5 & 27,1 & 66,9 \\
\hline Bolivia & Santa Cruz & 55,1 & 25,1 & 63,7 \\
\hline Chile & Coquimbo & 67,9 & 38,9 & 61,2 \\
\hline Chile & Santiago & 71,8 & 38,3 & 59,7 \\
\hline Chile & Valparaiso-Viña & 67,7 & 36,8 & 61,3 \\
\hline Costa Rica & Todo el país & 46,4 & 19,6 & 65,8 \\
\hline México & Monterrey & 50,5 & 18,7 & 58,5 \\
\hline Perú & Huancayo & 48,8 & 16,7 & 68,0 \\
\hline Perú & Lima & 55,9 & 20,2 & 63,4 \\
\hline Perú & Tarapoto & 44,7 & 16,2 & 80,3 \\
\hline Perú & Trujillo & 48,9 & 19,2 & 76,5 \\
\hline Venezuela & Todo el país & 20,2 & 6,8 & 69,4 \\
\hline \multicolumn{5}{|l|}{ Caribe } \\
\hline Antigua y Barbuda & Todo el país & 23,6 & 5,2 & 70,6 \\
\hline Bahamas & Todo el país & 30,9 & 7,8 & 77,3 \\
\hline Barbados & Todo el país & 36,3 & 10,8 & 60,4 \\
\hline Dominica & Todo el país & 37,1 & 13,0 & 52,4 \\
\hline Grenada & Todo el país & 23,3 & 10,2 & 71,4 \\
\hline Monserrat & Todo el país & 22,7 & 5,0 & 50,0 \\
\hline Guyana & Todo el país & 29,5 & 8,7 & 81,2 \\
\hline Jamaica & Todo el país & 33,8 & 15,2 & 68,1 \\
\hline Suriname & Área Urbana & 54,3 & 16,3 & 68,3 \\
\hline Trinidad y Tobago & Todo el país & 40,2 & 13,9 & 76,2 \\
\hline
\end{tabular}

dice no perseguir el mercado que representa los jóvenes de estas edades.

Finalmente, la asequibilidad de los productos tabáquicos es otra gran preocupación. Mientras que los países más desarrollados los productos alimenticios son mas asequibles que el tabaco, en los países menos desarrollados, incluidos todos los de América Latina, ocurre lo contrario. Como ejemplo comparativo se cita los precios de Marlboro y el Big Mac. Mientras que en Estados Unidos, Canadá o Suecia, el paquete de Marlboro es 2 y 3 veces más caro que un Big Mac, En la gran mayoría de los países de América Latina es el Big Mac es que es dos y tres veces más caro que el Marlboro.

\section{ALCOHOL}

\section{Consumo per capita de alcohol}

El consumo per capita de alcohol entre la población de 15 y más años de edad da una idea de la magnitud de los problemas ocasionados por el consumo crónico de bebidas alcohólicas, como la cirrosis hepática. Estos bebedores habituales suelen constituir una proporción relativamente baja (alrededor de 10\%) de todos los bebedores pero consumen la mitad del alcohol disponible. Existe información de consumo de alcohol de 30 países de las Américas que se presenta en el cuadro 3.

En 1996, los países productores de vino, Argentina principalmente, o sus vecinos eran los máximos consumidores de vino, mientras que Venezuela y los EUU lo eran de cerveza. Algunas naciones del Caribe, como Guyana y las Bahamas, eran los máximos consumidores de licores, que los convertían en los países con el mayor consumo per capita de alcohol de toda la región.

La información del cuadro recoge el consumo registrado de bebidas alcohólicas producidas legalmente. Sin embargo, en muchos países se producen grandes cantidades de bebidas alcohólicas de forma clandestina o para consumo casero. En Brasil, por ejemplo, se sabe que se producen anualmente alrededor de mil millones de litros de pinga que no se registra y cuyo consumo casi triplica la estimación de consumo per capita, elevándolo en 1996 hasta 14 litros de alcohol puro por persona.viii En Chile la producción clandestina supone un $20 \%$ de la legal y eleva el consumo per capita a 14,4 litros. ${ }^{9}$ En Ecuador, la producción clandestina triplica la legal y eleva el con- 
Cuadro 3. Consumo per capita registrado de litros de alcohol puro en población de 15 o más años de edad en 1996 en las Américas

\begin{tabular}{|c|c|c|c|c|}
\hline País & Total & Cerveza & Licores & Vino \\
\hline Antillas Neerlandesas & 8,8 & 4,6 & 3,4 & 0,8 \\
\hline Argentina & 9,6 & 2,1 & 0,4 & 7,1 \\
\hline Bahamas & 12,1 & 1,1 & 9,8 & 1,2 \\
\hline Barbados & 8,4 & 2,8 & 5,1 & 0,5 \\
\hline Belize & 5,8 & 2,6 & 3,1 & 0,1 \\
\hline Bolivia & 3,3 & 1,7 & 1,6 & 0,0 \\
\hline Brasil & 5,6 & 3,0 & 2,3 & 0,3 \\
\hline Canadá & 7,5 & 4,2 & 2,1 & 1,2 \\
\hline Chile & 7,1 & 2,4 & 2,0 & 2,7 \\
\hline Colombia & 6,4 & 4,3 & 2,1 & 0,0 \\
\hline Costa Rica & 5,7 & 0,9 & 4,7 & 0,1 \\
\hline Cuba & 3,5 & 1,0 & 2,3 & 0,2 \\
\hline Ecuador & 1,7 & 0,6 & 0,9 & 0,1 \\
\hline EEUU & 8,9 & 5,4 & 2,4 & 1,1 \\
\hline El Salvador & 2,5 & 1,3 & 1,2 & 0,0 \\
\hline Guatemala & 2,0 & 0,8 & 1,2 & 0,0 \\
\hline Guyana & 14,0 & 1,0 & 13,0 & 0,0 \\
\hline Haití & 6,6 & 0,0 & 6,5 & 0,0 \\
\hline Honduras & 2,4 & 1,2 & 1,2 & 0,0 \\
\hline Jamaica & 3,9 & 1,8 & 2,1 & 0,0 \\
\hline México & 5,0 & 4,1 & 0,9 & 0,0 \\
\hline Nicaragua & 2,3 & 0,5 & 1,8 & 0,0 \\
\hline Panamá & 5,7 & 3,4 & 2,2 & 0,1 \\
\hline Paraguay & 9,7 & 3,2 & 6,1 & 0,4 \\
\hline Perú & 4,0 & 1,6 & 2,2 & 0,2 \\
\hline República Dominicana & 5,9 & 2,1 & 3,7 & 0,1 \\
\hline Suriname & 4,7 & 3,1 & 1,6 & 0,0 \\
\hline Trinidad y Tobago & 3,7 & 1,5 & 2,1 & 0,1 \\
\hline Uruguay & 8,2 & 1,8 & 1,3 & 5,1 \\
\hline Venezuela & 9,4 & 5,8 & 3,5 & 0,1 \\
\hline
\end{tabular}

Fuente: Global Status Report on Alcohol.OMS,1999.WHO/HSC/SAB/99.11 0,0 significa consumo $<0,1$

sumo per capita a 8,4 litros de alcohol puro. Por tanto, los datos del cuadro 3 son subestimaciones del consumo real.

Los cuadros 4 y 5 indican que hay un ligero descenso en todo el mundo del consumo per capita de bebidas alcohólicas, expresado tanto en litros de bebidas como en kilogramos de alcohol puro. Mientras que el consumo en América Latina desciende ligeramente, en el Caribe y Norteamérica el consumo permanece estables y en algunas regiones del mundo, principalmente Asia, este consumo se incrementa. Sin embargo, la tendencia al descenso que se observa en América Latina y otras zonas del mundo es la consecuencia del incremento de la población adulta, ya que el consumo total de bebidas alcohólicas sigue aumentado. Entre 1998 y 1999 el consumo mundial de bebidas alcohólicas aumentó en 3 mil millones de litros hasta alcanzar 175 mil millones de litros en 1999.
Cuadro 4. Consumo per capita de bebidas alcohólicas en población de 15 y más años de edad por región del mundo

\begin{tabular}{|lrrrrrr|}
\hline \multicolumn{7}{c|}{ Litros per cápita } \\
\hline Área & 1990 & 1995 & 1996 & 1997 & 1998 & 1999 \\
\hline Total del mundo & 43,1 & 42,3 & 41,9 & 42,5 & 42,0 & 42,0 \\
Total Américas & 97,7 & 93,4 & 92,6 & 92,5 & 91,8 & 91,6 \\
Caribe & 32,8 & 34,1 & 34,4 & 34,0 & 34,6 & 34,5 \\
Norte América & 129,6 & 119,3 & 119,3 & 118,5 & 118,8 & 119,8 \\
Canadá & 110,1 & 99,3 & 98,2 & 97,8 & 99,7 & 100,2 \\
EEUU & 131,7 & 121,5 & 121,6 & 120,8 & 120,9 & 122,0 \\
América Latina & 76,1 & 77,9 & 76,6 & 77,2 & 75,9 & 74,8 \\
Argentina & 107,1 & 102,9 & 101,8 & 104,0 & 101,8 & 102,8 \\
Brasil & 77,6 & 86,5 & 84,5 & 83,8 & 82,1 & 80,5 \\
Colombia & 84,1 & 68,4 & 65,8 & 64,9 & 63,6 & 62,2 \\
México & 77,7 & 73,2 & 75,9 & 78,3 & 79,1 & 79,6 \\
Perú & 40,6 & 51,1 & 49,8 & 46,0 & 44,9 & 43,2 \\
Venezuela & 115,7 & 122,2 & 111,4 & 123,3 & 117,7 & 113,9 \\
Chile & 65,5 & 66,9 & 67,3 & 68,4 & 67,1 & 63,9 \\
Europa & 116,1 & 107,0 & 105,3 & 107,4 & 105,8 & 107,5 \\
Asia & 13,3 & 17,7 & 18,0 & 18,9 & 18,8 & 18,8 \\
África & 10,6 & 8,8 & 8,8 & 8,7 & 8,6 & 8,5 \\
Oceania & 146,2 & 124,2 & 121,6 & 119,5 & 120,0 & 120,4 \\
\hline
\end{tabular}

En América Latina se consumieron 24 mil millones de litros, 200 millones más que en 1998 y 4300 millones más que en 1990. En Norteamérica en 1999 se consumieron 29 mil millones de litros, 600 millones más que en 1998.

En el periodo 2001 a 2005 se espera que el consumo total de bebidas alcohólicas siga aumentando en todo el mundo excepto en la Unión Europea. Para 2005 se proyecta que se consumirán 190 mil millones de litros en todo el mundo. Para ese año se proyecta que en América Latina se consuman más de 25 mil millones de litros y en Norteamérica casi 32 mil millones. Este crecimiento será relativamente mayor en el consumo de cerveza que de otras bebidas alcohólicas. Se prevé que para 2005 el 84\% del volumen de bebidas alcohólicas consumidas será cerveza en América Latina y el Caribe, y casi el $86 \%$ en Norteamérica. No obstante, el consumo per capita de bebidas alcohólicas producidas legalmente tenderá a disminuir. Solo se proyecta que dos países de América Latina aumenten su consumo per capita: México y Chile. Sin embargo, es posible que si se tomase en cuenta la producción clandestina y casera, así como el mercado de contrabando el consumo per capita aumente en más países de la región. 
Cuadro 5. Consumo per cápita de bebidas alcohólicas expresado en kilogramos de alcohol puro en población de $\mathbf{1 5}$ y más años de edad por región del mundo

\begin{tabular}{|lrccccc|}
\hline \multicolumn{7}{|c|}{ Kgs de alcohol puro } \\
\hline Area & 1990 & 1995 & 1996 & 1997 & 1998 & 1999 \\
\hline Total del mundo & 4,3 & 4,2 & 4,2 & 4,3 & 4,1 & 4,1 \\
Total Américas & 8,5 & 7,5 & 7,4 & 7,3 & 7,3 & 7,2 \\
Caribe & 3,6 & 3,5 & 3,5 & 3,5 & 3,5 & 3,5 \\
Norteamérica & 9,9 & 9,1 & 9,0 & 8,9 & 9,0 & 9,1 \\
$\quad$ EEUU & 10,1 & 9,2 & 9,1 & 9,1 & 9,1 & 9,2 \\
Canadá & 8,1 & 7,4 & 7,4 & 7,5 & 7,7 & 7,8 \\
Latinoamérica & 7,7 & 6,6 & 6,5 & 6,4 & 6,3 & 6,1 \\
Argentina & 9,7 & 8,5 & 8,3 & 8,4 & 8,3 & 8,3 \\
Brasil & 10,6 & 8,3 & 8,2 & 8,0 & 7,7 & 7,5 \\
Chile & 6,1 & 6,3 & 6,3 & 6,4 & 6,3 & 6,0 \\
Colombia & 6,3 & 5,8 & 5,5 & 5,4 & 5,3 & 5,2 \\
México & 5,7 & 4,9 & 5,1 & 5,3 & 5,3 & 5,2 \\
Perú & 2,0 & 2,6 & 2,5 & 2,3 & 2,2 & 2,2 \\
$\quad$ Venezuela & 10,0 & 8,7 & 8,1 & 8,7 & 8,2 & 7,9 \\
Árica & 0,7 & 0,6 & 0,6 & 0,6 & 0,6 & 0,6 \\
Asia & 2,1 & 2,6 & 2,6 & 2,7 & 2,6 & 2,6 \\
Europe & 10,4 & 9,7 & 9,5 & 10,0 & 9,4 & 9,5 \\
Oceania & 9,9 & 8,5 & 8,5 & 8,5 & 8,7 & 8,9 \\
\hline
\end{tabular}

\section{Morbi-mortalidad atribuible al alcohol}

El alcohol ocasiona una pesada carga de enfermedad en las Américas. América Latina es la segunda región del mundo con mayor carga de enfermedad atribuible al alcohol después de los países más desarrollados del mundo, entre los que se incluye dos países de las Américas: Canadá y los EEUU. Mientras el $15,6 \%$ de la carga de enfermedad es atribuible al alcohol en los países más desarrollados, en América Latina esta cifra es del $14,7 \%$. La región del medio oriente es la que menor carga de enfermedad presenta con el $1 \%$.

\section{Prevalencia del consumo}

El cuadro 6 muestra la prevalencia de consumo de bebidas alcohólicas en los países de las Américas para los que se dispone de datos. La mayoría de estos sólo reportan prevalencia de consumo durante toda la vida. El porcentaje de hombres que han bebido alcohol alguna vez en su vida oscila entre el $60 \%$ de Haití y el 95\% de Canadá. Entre las mujeres estos porcentajes varían entre el $37 \%$ de México y el $90 \%$ de Colombia. En cualquier caso, los datos no son comparables entre países ya que la metodología de cada estudio es diferente. El cuadro 7 presenta datos de prevalencia de consumo entre jóvenes para cuatro países. Se observa una alta prevalencia de vida y entre el 20 y el $40 \%$ de estos jóvenes han consumido alcohol durante el último mes

\section{Desafíos para la prevención de los problemas rela- cionados con el consumo de alcohol}

Un primer desafío es reducir el consumo de bebidas de producción no controlada de manera que se garantice la salubridad de sus contenidos. El segundo es reducir la asequibilidad de estas bebidas. En este sentido, el precio de las bebidas alcohólicas legales determina la proporción de personas que las consumen. Los jóvenes son más sensibles a las subidas de precios que los adultos. La gran mayoría de los estudios sobre el tema indican que el aumento del precio de las bebidas alcohólicas mediante el incremento de los impuestos sobre su venta disminuye la proporción

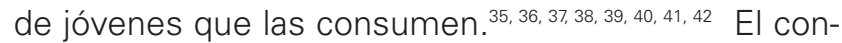
sumo disminuye tanto entre los bebedores más frecuentes como entre los ocasionales. El consumo entre las mujeres es más sensible a las variaciones del precio que entre los varones. Un estudio en los EEUU ha llegado a estimar que un incremento del $10 \%$ del precio reduciría en un $6,5 \%$ el numero medio de copas consumidas en un año. Por este motivo, unas de las políticas más eficaces para reducir el consumo de alcohol, sobre todo entre los jóvenes, es el aumento de los impuestos sobre la venta de bebidas alcohólicas. Los que se oponen a los incrementos de precio como medida de control del consumo de alcohol, sobre todo la industria del alcohol, aducen que la reducción de este consumo mediante incremento de precios o restricciones a su venta genera en los jóvenes una mayor demanda de otras drogas ilegales que sustituyen al alcohol. La mayoría de los pocos estudios realizados sobre este tema indican que esto no es cierto. Cinco estudios ${ }^{43,44,45,46}$ han demostrado que los jóvenes no sustituyen el alcohol por la marihuana cuando tienen menos acceso al alcohol. Un estudio ha demostrado que tampoco sustituyen al alcohol por heroína o cocaína. ${ }^{47}$

El tercer desafío es evitar la promoción de las bebidas alcohólicas. Los jóvenes de las Américas viven a diario una presión continuada y agresiva para iniciar el consumo de alcohol a edades tempranas y de forma excesiva. Esta presión se ejerce a través de los medios de propaganda tradicionales, pero sobre todo mediante el patrocinio de espectáculos deportivos y culturales, así como de otras técnicas promocionales cuyo último objetivo es las bebidas alcohólicas formen parte integral de la vida y hábitos de los jóvenes. Las respuestas actuales no han servido para controlar el marketing de productos alcohólicos. Esto unido a que la autorregulación de la propia industria alcoholera es inefectiva hace que la única intervención eficaz que tenemos son las medidas regulatorias y legislativas 
Cuadro 6. Prevalencia (\%) de consumo de alcohol en la población general de las Américas 1990-2000

\begin{tabular}{|c|c|c|c|c|c|c|c|}
\hline País & Año & Rango de Edad & Tamaño Muestra & Sexo & Alguna vez & Último año & Ultimo mes \\
\hline Argentina ${ }^{10}$ & 1996 & $\ldots$ & $\ldots$ & $\mathrm{T}$ & 66,7 & $\ldots$ & $\ldots$ \\
\hline Belizex"11 & 1993 & $\ldots$ & $\ldots$ & $T$ & 61,0 & $\ldots$ & $\ldots$ \\
\hline Bolivia $^{12}$ & $\ldots$ & $\ldots$ & $\ldots$ & $\begin{array}{l}H \\
M \\
T\end{array}$ & $\begin{array}{l}76,2 \\
60,4 \\
66,9\end{array}$ & $\ldots$ & $\ldots$ \\
\hline Canada $^{13}$ & 1995 & $>15$ & 10.385 & $\begin{array}{l}\mathrm{H} \\
\mathrm{M} \\
\mathrm{T} \\
\mathrm{T}\end{array}$ & $\begin{array}{l}95,2 \\
89,6 \\
92,3\end{array}$ & $\begin{array}{l}85,4 \\
78,8 \\
82,0\end{array}$ & $\begin{array}{l}80,6 \\
68,4 \\
25,6\end{array}$ \\
\hline Canada $^{14}$ & 1997 & $\ldots$ & $\ldots$ & $\begin{array}{l}\mathrm{H} \\
\mathrm{M} \\
\mathrm{T}\end{array}$ & $\begin{array}{l}78,1 \\
66,7 \\
72,3\end{array}$ & $\ldots$ & $\ldots$ \\
\hline Chile ${ }^{15}$ & 1996 & $\ldots$ & $\ldots$ & $\begin{array}{l}\mathrm{H} \\
\mathrm{M} \\
\mathrm{T}\end{array}$ & $\begin{array}{l}68,7 \\
53,6 \\
60,0\end{array}$ & $\ldots$ & $\ldots$ \\
\hline Colombia 16 & 1992 & $12+$ & $\ldots$ & $\begin{array}{l}\mathrm{H} \\
\mathrm{M}\end{array}$ & 90,6 & $\begin{array}{l}74,6 \\
74,9 \\
\end{array}$ & $\begin{array}{l}40,3 \\
39,8 \\
\end{array}$ \\
\hline Costa Rica $^{17}$ & 1995 & $12-70$ & 2.727 & $\begin{array}{l}\mathrm{H} \\
\mathrm{M} \\
\mathrm{T}\end{array}$ & $\begin{array}{l}82,0 \\
48,8 \\
65,3\end{array}$ & $\begin{array}{l}55,2 \\
29,6 \\
42,3\end{array}$ & $\begin{array}{c}18,4 \\
18,6 \\
17,2 \\
\end{array}$ \\
\hline Ecuador $^{18}$ & 1995 & $12-49$ & 3.501 & $\mathrm{~T}$ & 76,4 & 51,2 & \\
\hline EE.UU. ${ }^{19}, 20,21$ & 1995 & $\ldots$ & $\ldots$ & $\begin{array}{c}\mathrm{H} \\
\mathrm{M} \\
\mathrm{T} \\
\end{array}$ & $\begin{array}{l}85,8 \\
79,2 \\
82,3 \\
\end{array}$ & $\ldots$ & $\begin{array}{l}60,1 \\
45,0 \\
52,2\end{array}$ \\
\hline Guatemala $^{22}$ & 1990 & $\ldots$ & $\ldots$ & $\begin{array}{l}\mathrm{H} \\
\mathrm{M} \\
\mathrm{T}\end{array}$ & $\begin{array}{l}65,9 \\
48,3 \\
56,7\end{array}$ & $\ldots$ & $\ldots$ \\
\hline Haiti2 ${ }^{23}$ & 1991 & $\ldots$ & $\ldots$ & $\begin{array}{c}\mathrm{H} \\
\mathrm{M} \\
\mathrm{T}\end{array}$ & $\begin{array}{l}60,3 \\
56,4 \\
57,8\end{array}$ & $\ldots$ & $\begin{array}{l}\ldots \\
\ldots \\
\end{array}$ \\
\hline México ${ }^{24}$ & 1990 & $\ldots$ & $\ldots$ & $\begin{array}{c}\mathrm{H} \\
\mathrm{M} \\
\mathrm{T}\end{array}$ & $\begin{array}{l}73,0 \\
37,0 \\
54,0\end{array}$ & $\ldots$ & $\ldots$ \\
\hline México ${ }^{25}$ & 1989 & $12-65$ & 11.157 & $\begin{array}{l}\mathrm{H} \\
\mathrm{M} \\
\mathrm{T}\end{array}$ & $\begin{array}{l}87,9 \\
66,4 \\
76,5\end{array}$ & $\begin{array}{l}18,3 \\
30,6 \\
24,8\end{array}$ & $\begin{array}{l}21,6 \\
23,6 \\
22,7\end{array}$ \\
\hline Panamá ${ }^{6}$ & 1992 & $\ldots$ & $\ldots$ & $\begin{array}{c}\mathrm{H} \\
\mathrm{M} \\
\mathrm{T} \\
\end{array}$ & $\begin{array}{l}72,1 \\
37,9 \\
54,2\end{array}$ & $\ldots$ & $\ldots$ \\
\hline Paraguayy ${ }^{27}$ & 1997 & $\ldots$ & $\ldots$ & $\begin{array}{c}\mathrm{H} \\
\mathrm{M} \\
\mathrm{T}\end{array}$ & $\begin{array}{l}88,0 \\
75,0 \\
79,5\end{array}$ & $\ldots$ & $\ldots$ \\
\hline Peru ${ }^{28}$ & 1993 & $\ldots$ & $\ldots$ & $T$ & 82,9 & $\ldots$ & $\ldots$ \\
\hline República Dominicana ${ }^{29}$ & 1992 & & $\mathrm{H}$ & $\begin{array}{c}64.8 \\
M \\
T\end{array}$ & $\begin{array}{l}46,0 \\
55,0\end{array}$ & $\ldots$ & $\ldots$ \\
\hline Venezuela ${ }^{30}$ & 1997 & $\ldots$ & $\ldots$ & $\mathrm{T}$ & 62,9 & $\ldots$ & $\ldots$ \\
\hline
\end{tabular}




\section{Cuadro7. Prevalencia de consumo de alcohol en población de jóvenes y adolescentes en las Américas 1990-2000}

\begin{tabular}{|c|c|c|c|c|c|c|c|}
\hline País & Año & Rango de Edad & Tamaño Muestra & Sexo & Alguna vez & Último año & Ultimo mes \\
\hline Brasi $^{31}$ & 1997 & $10-18$ & 15.501 & T & 67,6 & 62,3 & 34,6 \\
\hline Belize $^{32}$ & 1998 & $12-18$ & 1.527 & $\begin{array}{l}\mathrm{H} \\
\mathrm{M} \\
\mathrm{T}\end{array}$ & $\begin{array}{l}58,8 \\
49,0 \\
53,5\end{array}$ & 35,4 & 22,5 \\
\hline Chile $^{33}$ & 1995 & escolares. & 29. 066 & $\begin{array}{l}\mathrm{H} \\
\mathrm{M} \\
\mathrm{T}\end{array}$ & $\begin{array}{l}76,2 \\
69,5 \\
72,7\end{array}$ & 62,7 & 3,5 \\
\hline EE.UU. ${ }^{34}$ & 1996 & $\ldots$ & $\ldots$ & $\begin{array}{l}H \\
M \\
T\end{array}$ & $\begin{array}{l}70,0 \\
60,2 \\
64,9\end{array}$ & $\ldots$ & $\ldots$ \\
\hline
\end{tabular}

necesarias para asegurar que la gente joven no esté expuesta a los mensajes promocionales sobre el alcohol.

\section{Drogas ilegales}

La producción, el tráfico y el consumo de drogas en las América crea violencia, enfermedad y muerte y afecta la gobernabilidad de los países de la región. Al mismo tiempo, el problema de las drogas exacerba la propia marginación social y la pobreza que la genera.

\section{Cultivo y Producción}

América Latina produce la totalidad de los precursores (hoja y pasta base) de la cocaína del mundo. Perú, Colombia y Bolivia producen el $98 \%$ de la hoja de coca de todo el mundo. Estos tres, son los países de América Latina y el Caribe que tienen más del 1\% de la superficie arable dedicada a cultivos ilícitos. Entre 1994 y 1998 la superficie dedicada al cultivo de la coca en los países andinos parece haber disminuido en un $17 \%$, pero el rendimiento de los cultivos ha permanecido básicamente estable.

Se estima que en 1996 se produjeron 300.000 toneladas de hoja de coca de las cuales se extrajeron 1.000 toneladas de cocaína. En Bolivia la actividad económica relacionada con la economía de la coca genera unos 135.000 empleos, es decir, el 6,4\% del empleo del país. ${ }^{48}$ El $65 \%$ de la producción de cocaína llega a los mercados de Europa. El resto se destina a los EEUU. Este contrabando tiene efectos perniciosos sobre la economía de los países de la región. La infraestructura del tráfico de drogas sirve para el contrabando de otros muchos bienes que escapan del control fiscal, en países donde una parte importante de la recaudación de impuestos se produce a través de la recolección de impuestos sobre la venta legal de productos.
Alrededor del $90 \%$ del cultivo y producción de opiáceos procede de Asia, fundamentalmente Afganistán y Myanamar. En América Latina existe un creciente cultivo de amapola fundamentalmente en México y Colombia. En este último país la superficie dedicada al cultivo de la amapola creció un 17\% de 1996 a 1998. En 1998, se estima que existían 7.350 hectáreas dedicadas al cultivo de la amapola frente a las 78.200 dedicadas a la coca. En todo caso, la producción de heroína en América Latina y el Caribe es un porcentaje muy bajo de las más de 300 toneladas que se producen en todo el mundo anualmente.

Se ha observado un incremento de la superficie dedicada al cultivo de Cannabis, que se consume en forma de marihuana. En Colombia, por ejemplo, hay 5.000 hectáreas dedicadas al cultivo de esta planta. En este país se estima que se han destruido entre 850.000 y un millón de hectáreas de bosque para implantar cultivos de coca, Cannabis y amapola entre 1974 y 1998.

En las Américas se producen drogas sintéticas. Las principales son los estimulantes anfetamínicos, que incluye la anfetamina, la metanfetamina y, más recientemente la metilenodioximetanfetamina o éxtasis. Si la detección de laboratorios ilegales de drogas sintéticas es un indicador de los lugares de producción, la mayoría de ellos han sido detectados en Estados Unidos, Polonia y Colombia.

El mercado de drogas ilegales mueve un total de 600 mil millones de dólares anuales en América Latina y el Caribe ${ }^{49}$ La economía de muchos países americanos depende de este mercado, y en algunos casos es el factor más importante de esta economía. 


\section{Consumo}

La demanda de drogas ha sido estimulada por la proximidad a los centros de producción y sirve, en muchos casos, para mantener mercados locales de consumo que financian las infraestructuras del contrabando con destino a mercados mayores. Aunque no hay suficiente información, se tiene la impresión de que el consumo está incrementándose, especialmente de sustancias sintéticas.

La marihuana es la droga ilegal de mayor consumo en el mundo y en las Américas. Se estima que existen alrededor de 45 millones de consumidores en las Américas (cuadro 8).

\section{Cuadro 8. Prevalencia de consumo de marihuana al menos una vez en el último año en la década de los 90 , por región del mundo}

\begin{tabular}{|lcc|}
\hline Región & $\begin{array}{c}\text { Prevalencia } \\
\text { (millones de usuarios) }\end{array}$ & Tasa de Prevalencia (\%) \\
\hline África & 25,3 & 3,4 \\
Asia & 50,9 & 1,5 \\
Europa & 16,3 & 2,2 \\
América Latina y el Caribe & 26,3 & 5,3 \\
América del Norte & 19,2 & 6,5 \\
Oceanía & 3,2 & 11.2 \\
\hline Total & 141,2 & 2,4 \\
\hline
\end{tabular}

La proporción de la población latinoamericana que ha consumido marihuana alguna vez en su vida varía entre el $2 \%$ de Paraguay y República Dominicana y el $17 \%$ de Chile. ${ }^{50}$ En los EEUU esta prevalencia de vida sube hasta el 35\% (Cuadro 9). La cocaína es la segunda droga más consumida. En la mayor parte de los países entre el 1 y el $4 \%$ de la población ha consumido cocaína alguna vez en la vida. ${ }^{51}$ En los EEUU esta cifra es de alrededor del $11 \%$. Este consumo, no obstante, se concentra en los adolescentes y adultos jóvenes de menor nivel socioeconómico. Entre los adolescentes la prevalencia de uso habitual de cocaína puede alcanzar entre el 1 y el $5 \%$.

\section{Impacto social y de salud del consumo de drogas}

Además de la adicción, el consumo de drogas es un factor determinante de la transmisión del SIDA y de otras enfermedades infecciosas, especialmente en el cono sur del continente. Este consumo en las embarazadas también está asociado a déficits físicos y mentales del desarrollo fetal y del recién nacido. El consumo de drogas agrava, de esta manera, los problemas infecciosos e infantiles de las capas socioeconómicas con menos capacidad para hacerles frente.

El consumo de drogas tiene un impacto que rebasa el campo de la salud. En el campo de la educación es responsable de una alta tasa de abandono escolar. En Chile, por ejemplo, el $20 \%$ de los escolares han consumido drogas ilegales en los últimos 12 meses. Muchos de ellos, especialmente los que pertenecen a familias menos acomodadas, no llegan a terminar la primaria. En Perú, la cifra de fracaso escolar en primaria es del $21 \% .{ }^{52}$ Tras abandonar la escuela, muchos de estos jóvenes, ya adictos, se mantienen vendiendo droga, entrando en la cultura de las pandillas violentas como mecanismo de apoyo social, que solo ahonda la marginación y la pobreza en la que viven. En El Salvador, casi la mitad de los pandilleros consumen drogas ilegales a diario..$^{53}$

\section{La contribución del sector salud a la solución del problema de las drogas}

Doce países de America Latina tienen Planes Nacionales de Control de Drogas. Estos planes hacen énfasis en el control de la oferta mediante la destrucción de los cultivos, la acción policial sobre el tráfico y la criminalización del consumo. Los resultados hasta ahora de este enfoque han sido limitados. Es necesario hacer hincapié en el control de la demanda, mediante intervenciones de prevención, desintoxicación, tratamiento y rehabilitación, así como de reducción de daño. Para muchos adictos, el tratamiento y la reinserción son la únicas opciones que tienen para salir de la espiral de marginación, violencia y pobreza. Esta es una contribución fundamental del sector salud.

\section{CONCLUSIÓN}

Aunque el consumo de sustancias adictivas constituye una amenaza obvia para la salud pública, muchos gobiernos están lejos de adoptar medidas contundentes para reducir su consumo. En algunos casos se debe a una subestimación del problema, en otros casos a cierta resignación frente a la dificultad que entraña su abordaje. En el caso de las drogas ilegales ha habido énfasis en las intervenciones para reducir la oferta en detrimento de las intervenciones para reducir la demanda, especialmente las que implican al sector salud. En el caso de las drogas legales, como el tabaco y el alcohol, muchos gobiernos dudan en actuar por temor a que el control de dichas sustancias tenga consecuencias económicas y políticas indeseables.

Los ejemplos de países que han sido efectivos en atajar el consumo de estas sustancias legales demuestran que no existen las consecuencias económicas negativas pregonadas por las respectivas industrias manufactureras. Por ejemplo, un estudio del Banco Mundial indicaba que sólo dos países de todo el mundo, Zimbawe y Malawi, tienen economías lo 


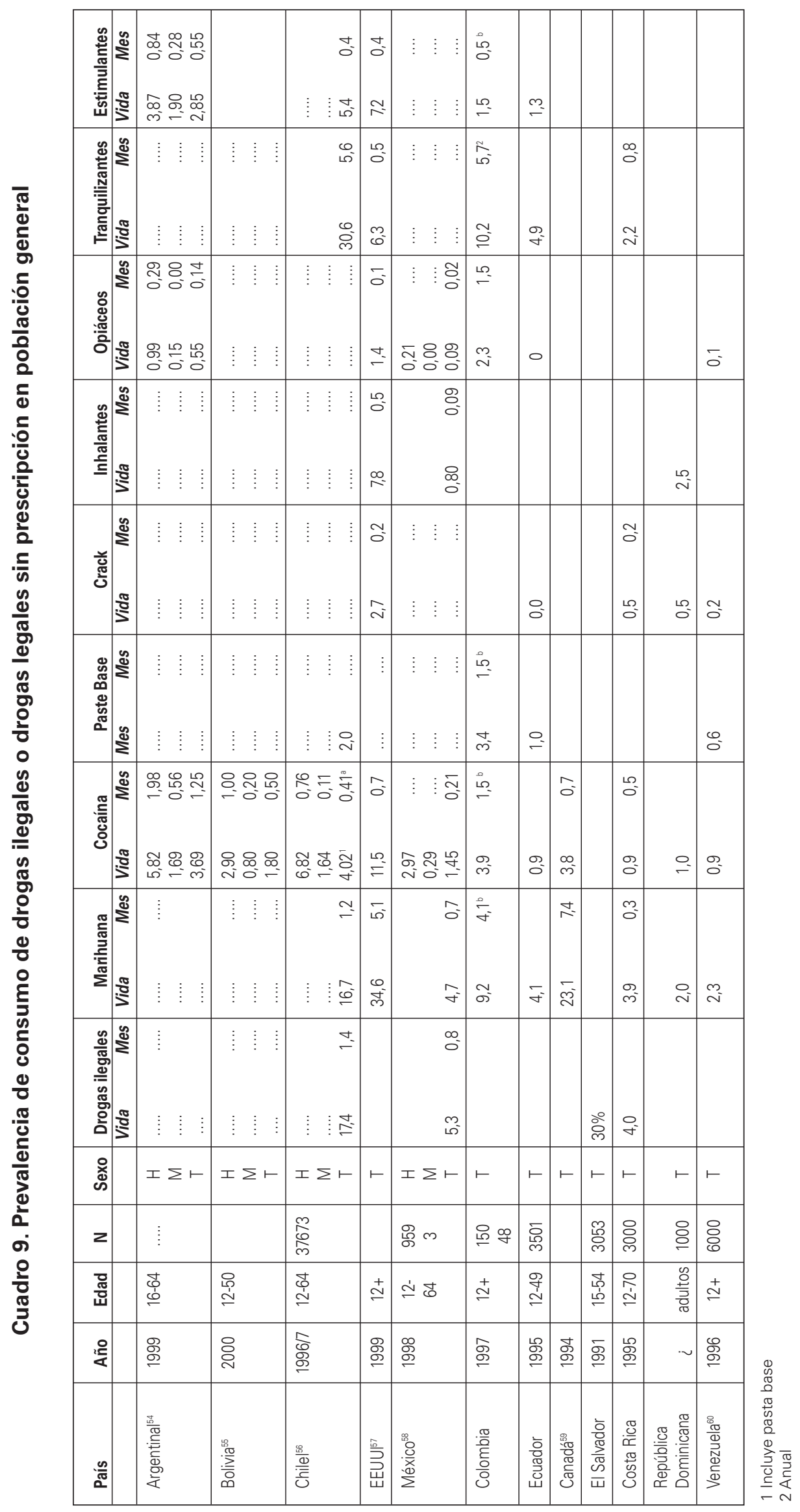


suficientemente dependientes del tabaco como sufrir algún efecto negativo si desapareciese el cultivo del tabaco, circunstancia esta que no se producirá de inmediato ni aún con la aplicación de las políticas más restrictivas del tabaquismo.

Aunque la responsabilidad gubernamental es amplia a la hora de aplicar soluciones a los problemas que plantean el consumo de drogas legales e ilegales, no es menos importante el papel que debe jugar la sociedad civil en su conjunto. Las sociedades de profesionales de la salud, padres y jóvenes, educadores y educandos, sindicatos y patronos, deben convertirse en abogados de las medidas de política social y económica necesarias para reducir la lacra de las drogas en nuestra sociedad. Todos tiene algo que aportar a la generación de la voluntad política necesaria. Todos tienen algo que ganar en este empeño ya que, aunque las medidas de control de estas sustancias requieren una voluntad política decidida, los costos políticos, en última instancia, son menores de lo que los adversarios de la salud publica quieren hacernos creer o tratan de infligir. Y en todo caso, mucho menores que los beneficios para la salud de las poblaciones.

\section{BIBLIOGRAFÍA}

(1) Departamento de Salud y Servicios Sociales de los EEUU. Tabaquismo y Salud en las Américas: Informe de la Cirujana General, 1992, en colaboración con la OPS. Atlanta, Georgia, DHHS, servicio de salud publica. Centros para el control de las enfermedades: Oficina de tabaquismo y Salud; 1992; DHHS, publicación No. (CDC) 92-8420.

(2) Organización Panamericana de la Salud. El tabaquismo en América Latina, Estados Unidos y Canadá: período 1990-1999. OPS, Washington DC, 2001.

(3) Rojas M, Barnett B, Peruga A, Selin. El tabaquismo en el Caribe Inglés. Boletin Epidemiologico, 2001.

(4) Warren, W.; Riley, L.; Asma, S.; Eriksen, M.; Green, L.; Blanton, C.; Loo, C.; Batchelor, S.; Yach, D. (2000) Tobacco use by youth: a surveillance report from the global youth tobacco survey. Geneva: World Health Organization.

(5) Rojas M, Barnett B, Selin H, Peruga A. Encuesta mundial sobre el tabaco en la juventud: estado de su ejecución en américa latina y el caribe. Boletín Epidemiológico.

(6) Organización Mundial de la Salud. Encuesta mundial de tabaquismo en los jóvenes. Datos no publicados.

(7) Organización Panamericana de la Salud. Resolución del 43er Consejo Directivo de la OPS. Septiembre 2001.

(8) Dunn J y LaranjeiraR. Memorandum a la Organización Mundial de la Salud, Ginebra, 15 de noviembre de 1996

(9) Organización Panamericana de la Salud. Special report. Epidemiological report on the use and abuse of psychoactive substances in 16 countries of Latin America and the caribbean. Bulletin of PAHO 1990, 24(1) 97-139
(10) Matos E, et al. 1996. Epidemiological studies of chronic diseases in developing countries. Study funded by Clinical Trail Service Unit (CTSU), Oxford, U. K. April 1996.

(11) Pride Belize Survey Project Team. 1993. Survey of Drug Prevalence and Attitudes in Belize City. August 1993, Belize

(12) Del Castillo FA, Salinas NF. UNDATED. El uso indebido de drogas en Bolivia estudio comparativo (poblacion urbana 1992-1996). Centro Latinoamericano de Investigacion Cientifica. Celin, Bolivia

(13) Williams, B, Single, E, McKenzie, D. 1995. Canadian Profile. Canadian Centre on substance Abuse Addidtion Research Foundation of Ontario: Toronto, Ontario

(14) Williams, B, Single, E, McKenzie, D. 1997. Canadian Profile. Canadian Centre on substance Abuse Addidtion Research Foundation of Ontario: Toronto, Ontario.

(15) Sistema Nacional de Información Sobre Drogas, Chile. 1996. Estudio Nacional de consumo de Drogas: Informe Final 1996. CONACE, Ministerio del Interior. Santiago, Chile, Mayo de 1996.

(16) Ramírez, LFD, Ospina, ER, Rivero, DC, Campos, JH. 1992. Consumo de Sustancias Psicoactivas llegales en Colombia. Centro de Investigación y Estudios Internacionales sobre Droga (CIDNE): Santa Fe de Bogota, DC.

(17) WHO. 2001. Survey of drinking patterns and problems in seven developing countries

(18) CONSEJO Nacional de Control de Sustancias Estupefacientes y Psicotropicas (CONSEP). Segunda Encuesta Nacional sobre Consumo de Drogas Ecuador, 1995. Direccion de Asesoria Tecnica Sistema Ecuatoriano de Vigilancia Intergral Para la Prevencion de las Drogas: Embajada de Los Estados Unidos De America, NAS.

(19) National Institute on Alcohol Abuse and Alcoholism (NIAAA). 1995. Quick facts: Projected numbers of alcohol abusers, alcoholics, and alcohol abusers and alcoholics combined, 1985, 1990, 1995. Pre-generated July 2001. http://www.niaaa.nih.gov/databases/abdep1.txt

(20) National Institute on Alcohol Abuse and Alcoholism (NIAAA). 1995. Quick facts: Percent reporting alcohol use in the past month by age group and demographic characteristics: NHSDA, 1994-97. Pre-generated July 2001. http://www.niaaa.nih.gov/databases/dkpat4.txt.

(21) National Institute on Alcohol Abuse and Alcoholism (NIAAA). 1995. Quick facts: Percent reporting heavy alcohol use in the past month by age group and demographic characteristics: NHSDA, 1994-97. Pre-generated July 2001. http://www.niaaa.nih.gov/databases/dkpat2.txt

(22) Development Associates, Inc. 1990. Drug awareness needs assesment for Guatemala: final report. Narcotics awareness and Education Project. Submitted to: Office of Human Resource Development, Guatemala. 29 November 1990

(23) Development Associates, Inc. 1991. National study of drug prevelence and attitudes toward drug use in Hati: revised final report. Narcotics awareness and Education Project Submitted to: the Association for the Pre- 
vention of Alcoholism and Other Chemical Dependencies. Port-au-Prince. 14 June 1991.

(24) Medina-Mora ME. 1990. Epidemiologic status of drug abuse in Mexico. Bulletin of PAHO. 24(1).

(25) Secreataría de Salud (1989) Primera encuesta nacional de adicciones. Secretaría de salud. Dirección General de Epidemiología, Instituto Mexicano de Psiquiatría. México

(26) Jutkovitz JM, et al. 1992b. Survey on drug prevalence and attitudes in the urban Panama. Narcotics awareness and education project.

(27) Míguez HA, Pecci MC. 1997. The epidemiology of drug and alcohol abuse in Paraguay. Comite Paraguay-Kansas. Agency for International Development. Development Associates, Inc. November 1997.

(28) Ortega, J. 1993. Drunk driving in Peru, a staistical and sociological analysis. Paper presented to the 19th Annual Alcohol Epidemiology Symposium of the Kettil Brunn Society for Social and Epidemiological Research on Alcohol, Krakow, Poland, 7-11 June 1993.

(29) Jutkovitz JM, et al. 1992a. Survey on drug prevalence and attitudes in the Dominican Republic, Arlington. Narcotics awareness and education project.

(30) Consejo Nacional de Drogas CND. 1997 National Household Survey 1996, Preliminary Report. Caracas, Conseil Nacional de Drogas.

(31) CEBRID.IV levantamiento sobre o uso de drogas entre estudantes de 1 e 2 graus em 10 capitais brasileriras .1997

(32) National Drug Abuse Control Council (NDACC). 1998. Preliminary Report of School Study Consumption of Alcohol, Tobacco and Drugs in Belize City and Dangriga. Belize

(33) Caris, L. 1996. Primer Informe De Consumo de Alcohol, Tabaco Y Drogas en Escolares Del Pais En 1995. Santiago, Chile.

(34) United States (US) Department of Health and Human Services. 1996. National survey results on Drug Use from The Monitoring the Future Study, 1975-1995. Vol 1, Secondary school student. Washington, DC

(35) Chaloupka FJ, Wechsler H. The impact of price, availability, and alcohol control policies on binge drinking in college. NBER working paper Series no. 5319, Cambridge, Mass: National Bureau of Economic Research, 1995.

(36) Coate D, Grossman M. Effects of alcoholic beverage prices and legal drinking ages on youth alcohol use. J. of Law and Economics, 1988; 31:145-171

(37) Grossman M, Coate D, Arluck G. Price sensitivity of alcoholic beverages in the USA. En MH Moore y DR Gerstein (eds) Alcohol and Public Policy: beyond the shadow of prohibition. Washington DC: National Academy, 1987

(38) Grossman M, Chaloupka FJ, Sirtalan I.An empirical analysis of alcohol addiction: results from the monitoring the future panels. Economic Inquiry, 1998; 36: 3948
(39) Kenkel DS (1993) Drinking, driving and deterrance: the social costs of alternative policies. J of Law and Economics, 1993; 36:877-914

(40) Laixuthai A, Chaloupka FJ. Youth alcohol use and public policy. Contemporary issues, 1993; 11:69-81

(41) Sutton M, Godfrey C. A grouped data regression approach to estimating economic and social influences on individual drinking behavior. Health Economics, 1995; 4:237-247

(42) Yamada T, Kendix M, Yamada T. the impact of alcohol consumption and marijuana use on high school graduation. Health Ecpnomics, 1996; 5:77-92

(43) Farrely M, Bray J, Zarkin G, Wendling B, Pacula R. The effects of prices and policies on the demanda for marijuana: evidence from the national household surveys on drug abuse. NBER working paper 6940, National Bureau fo Economic Research: Cambridge, Mass, 1999

(44) Pacula R. Does increasing the beer tax reduce consumption, J of Health Economics 1998; 17: 557-586

(45) Pacula R. Adolescent alcohol and marijuana consumption: is there really a gateway effect? NBER working paper WP801, Berkeley, CA: prevention research center, 1998

(46) Thies C, Register C. Decriminalization of marijuana and the demand for alcohol, marijuana and cocaine. The Social Science Journal, 1993; 30:

(47) Saffer H, Chaloupka FJ. Demographic differentials in the demand for alcohol and illicit drugs. En Chalupka FJ et al. (eds) The economic anlysis of substance use and abuse, Chicago: National Bureau of Economic Research, 187-211. 1999

(48) Comisión Económica Para América Latina. Panorama social de América Latina, edición 1999-2000, LC/G.2068-P. Santiago de Chile, Julio 2000

(49) Arraigada I., Hopenhayn M. Producción, tráfico y consumo de drogas en América Latina. Serie políticas sociales. CEPAL, Chile 2000.

(50) Naciones Unidas. Comisión de Estupefacientes, doc. C/CN.7/1999/CPR.5, febrero 1999

(51) OPS. La salud en las Américas. Washington 1998

(52) Programa internacional de control de drogas de las Naciones Unidas. Informe Mundial sobre Drogas. Oxford University Press, 1997

(53) OPS. La violencia de las pandillas en El Salvador. Pendiente de publicación

(54) Miguez H. (1999) Estudio nacional sobre sustancias adictivas. Población nacional urbana. Buenos Aires. Secretaría de Programación para la Prevención de la Drogadicción y la Lucha contra el Narcotráfico

(55) Alcaraz F, Soliz RM, Zuazo J. (2000) El uso indebido de drogas en ciudades bolivianas: Estudio urbano, año 2000. Centro Latinoamericano de Investigación Científica (CELIN) Bolivia.

(56) Consejo Nacional para el Control de Estupefacientes (1998). Tercer Estudio Nacional para el Consumo de Drogas en Chile. CONACE

(57) SAMHSA (1999) National Household Survey on Drug Abuse. 
(58) Secretaría de Salud. Encuesta Nacional de Adicciones, 1998. Mexico

(59) Canadian Profile 1999: Alcohol, Tobacco and Other Drugs. Canadian center for Substance abuse
(60) Citado en CICAD/OEA BID Sistema interamericano de datos uniformes sobre consumo de drogas. Primer informe SIDUC 2000 